\title{
New Formulation of Equation of State and Study of Elastic Properties of Alkaline Earth Oxides under High Pressure
}

\author{
S.P. SingH ${ }^{a, *}$ AND D. SingH ${ }^{a, b}$ \\ ${ }^{a}$ Department of Physics, Dr. B.R. Ambedkar Govt. Degree College, \\ Mainpuri (UP)-205001, India \\ ${ }^{b}$ Department of Physics, R.B.S. College, Agra (UP)-282007, India
}

Received: 14.05.2021 \& Accepted: 20.06.2021

Doi: 10.12693/APhysPolA.140.131 *e-mail: singh525sps@yahoo.com

\begin{abstract}
A new analytical equation of state based on the Eulerian finite strain scheme has been formulated. The $n$-th power of edge length by compression has been used. This equation correctly evaluates models of the high-pressure theory and corresponds well to the experimental data of geophysical minerals. The new equation of state is capable of producing other prominent equations of state such as the BirchMurnaghan equation of state, the third-power Eulerian equation of state, etc. The derived equation has been used to develop the expression of the pressure dependence of bulk modulus and its pressure derivative. The developed expressions have been used to test and investigate the pressure dependence of bulk modulus and its pressure derivative of alkaline earth oxides viz. $\mathrm{MgO}, \mathrm{CaO}$. It is found that the computed results using the present models show a better agreement with available experimental results. It is concluded that new expressions are capable of predicting the elastic properties of earth's minerals under high-pressure conditions. It can be used in geophysical applications.
\end{abstract}

topics: equation of state (EOS), $n$-th power of edge length, pressure dependence of bulk moduli, high pressure

\section{Introduction}

Currently, understanding the state of earth's interior minerals and its density distribution under high pressure and high temperature attracts many researchers. Mineral physics has played a major role in explaining the physical properties of matter under extreme conditions of pressure and temperature. The results of such researches have a significant impact in the fields of geophysics, materials science and condensed matter physics. Since compressibility is defined by the derivative of volume, therefore elasticity offers more information to interpret the pressure and temperature dependence of the equation of state (EOS) [1]. The elastic moduli are appropriate physical quantities to depict the mechanical properties of materials. They are utilized to assess the elastic strains or energies in materials under the pressure of different roots: outside, inside, warm, and so on. The elastic properties are directly connected with numerous key physical quantities, for example, seismic velocities, anisotropy, Cauchy's deviation, thermal conductivity, the Debye temperature, interatomic potentials, etc. The behaviors of elastic moduli under the effect of high pressure and high temperature have aroused great interest in experimental [2-4], as well as theoretical researchers [5-12].
This paper is divided into two parts. The first part deals with the development of a new equation of state depending upon the Eulerian finite strain scheme to utilize the $n$-th power of the edge length by pressure. The Eulerian finite strain has to be truncated for this purpose. We have derived the second-order and third-order equation of states. The second part deals with the derivation of the pressure dependence of isothermal bulk modulus and its pressure derivatives with the help of the proposed equations of state. The derived expressions will be applied to alkaline earth oxides viz. $\mathrm{MgO}$, $\mathrm{CaO}$. Finally, the validity and applicability of the proposed work will be tested and evaluated under extreme pressure.

Note that the study of alkaline earth oxides (AEOs) is of great interest for several reasons. These oxides have been considered a typical case in understanding bonding in ionic oxides [13]. Further, they are one of the most fundamental materials for industrial science. These oxides are also a major constituent of earth's lower mantle (between 600 and $2900 \mathrm{~km}$ in depth) [5]. The electronic structure [14], structural phase transitions [6-9], elasticity $[1-12]$, thermal properties $[1-3,8,9]$, stability and the equation of state $[10,11]$ of these oxides have been extensively studied theoretically [5-10] as well as experimentally [1-4]. 
The results of the proposed study are discussed and the comparison is made with the available experimental data and other theoretical methods. The utility of the present study under extreme pressure is essential as it allows the extrapolation into the regions for which the experimental data are not available abundantly.

\section{Theoretical analysis}

Let us assume that a unit cubic body has the length of an introductory edge $X_{0}$, and its volume $V_{0}=X_{0}^{3}$. If the unit cubic body is uniformly compacted along the edge length $X$, the volume of such body is $V=X^{3}$. The body displacement $u$ $(u<0)$ due to compression $V / V_{0}$ can be represented in terms of the introductory edge length, and thus the length change can be given as

$$
X_{0}=X-u \text {, }
$$

where the displacement $u(u<0)$ due to compression can be represented in terms of the introductory edge length.

The $n$-th power of the change in length by compression, i.e.,

$$
\begin{gathered}
X^{n}-X_{0}^{n} \equiv X^{n}-(X-u)^{n}= \\
X^{n}-X^{n}\left(1-\frac{u}{X}\right)^{n},
\end{gathered}
$$

can be expanded, neglecting the higher terms of $u$ in the term $\left(1-\frac{u}{X}\right)^{n}$, because $u<0$. Then, (2) takes the following form:

$$
X^{n}-X_{0}^{n}=n u X^{n-1}-\frac{n(n-1)}{2} u^{2} X^{n-2} .
$$

Due to uniform compression, the displacement will be proportional to the reference edge length

$$
u=c X
$$

where $c$ is a proportional constant at a given compression, referred to as the strain in linear elasticity. Now, using (3) and (4), we have

$$
X^{n}-X_{0}^{n}=\left(n c-\frac{n(n-1)}{2} c^{2}\right) X^{n} .
$$

The finite strain under the Eulerian scheme $\varepsilon_{E}$ is defined as:

$$
\varepsilon_{E} \equiv c-\frac{n-1}{2} c^{2} \text {. }
$$

Hence, from (5) and (6), we get

$$
X^{n}-X_{0}^{n}=n \varepsilon_{E} X^{n} .
$$

Thus, we can easily write the finite strain for the $n$-th power of the edge length.

The body is compressed if $\varepsilon_{E}$ is negative. With the use of (7), one can obtain the Eulerian finite strain $f$, expressed in terms of $\frac{V_{0}}{V}$. Namely,

$$
f=-\varepsilon=\frac{1}{n}\left[\left(\frac{V_{0}}{V}\right)^{n / 3}-1\right],
$$

where $V_{0}$ is the volume at $P=0$, and

$$
\frac{V_{0}}{V}=(1+n f)^{\frac{3}{n}} \text {. }
$$

The partial derivative of this Eulerian finite strain $f$ with respect to volume is given as follows:

$$
\frac{\partial f}{\partial V}=-\frac{1}{3 V_{0}}(1+n f)^{\frac{3}{n}+1} .
$$

In the compressed state, the Helmholtz free energy of a matter increases. Thus, the Helmholtz free energy can be expressed in the form of the Taylor series expansion of the Eulerian finite strain

$$
A=a_{0}+a_{1} f+a_{2} f^{2}+a_{3} f^{3}+a_{4} f^{4}+\ldots
$$

\subsection{Second-order equation of state}

To obtain the second-order equation of state (EOS), (11) has to be truncated up to the second term. Hence, (11) reduces to

$$
A=a_{0}+a_{1} f+a_{2} f^{2} .
$$

The first coefficient in (12) should be taken as $a_{0}=0$ because pressure in the uncompressed condition case (when $V=V_{0}$ and $A=0$ ) is zero. We get then

$$
A \cong a_{1} f+a_{2} f^{2} .
$$

In isothermal EOS, the pressure $P$ is expressed as a function of the volume $V$. From the thermodynamics identity, the pressure is the volume derivative of the Helmholtz energy $A$, i.e.,

$$
P=-\left(\frac{\partial A}{\partial V}\right)_{T}
$$

and the relation between pressure and volume becomes

$$
P=\frac{\left(a_{1}+2 a_{2} f\right)}{3 V_{0}}(1+n f)^{\frac{3}{n}+1} .
$$

Under the uncompressed condition, the first coefficient in (15) is taken as $a_{1}=0$ because pressure should be zero then. Since $P=0$ and $V=V_{0}$, (15) reduces to the form of

$$
P=\frac{2 a_{2} f}{3 V_{0}}(1+n f)^{\frac{3}{n}+1} \text {. }
$$

Now, the value of the second coefficient $a_{2}$ can be easily determined using the thermodynamic relation for isothermal bulk modulus, i.e.,

$$
K_{T}=-V\left(\frac{\partial P}{\partial V}\right)_{T} .
$$

The partial derivative of pressure with respect to the volume in the uncompressed state (when $P=0$ and $\left.V=V_{0}\right)$ results in $f=0$. Therefore

$$
\left(\frac{\partial P}{\partial V}\right)_{T, P=0}=-\frac{K_{T_{0}}}{V_{0}},
$$

where $K_{T_{0}}$ is the isothermal bulk modulus at standard temperature.

Using (16)-(18) and assuming $P=0$, one gets the coefficient $a_{2}$ :

$$
a_{2}=\frac{9}{2} K_{T_{0}} V_{0}
$$

Substituting (19) into (16), the $P-V$ relation is obtained as

$$
P=3 f K_{T_{0}}(1+n f)^{\frac{3}{n}+1} .
$$


The above result (20) is the second-order EOS, given in terms of the Eulerian finite strain $f$ and $K_{T_{0}}$ - the isothermal bulk modulus at standard temperature.

Next, using (17), we obtained the expression for isothermal bulk modulus. It has the following form:

$$
K_{T}=K_{T_{0}}(1+n f)^{\frac{3}{n}+1}[1+(3+2 n) f]
$$

and it is given in terms of the Eulerian finite strain $f$.

The first pressure derivative of isothermal bulk modulus is defined as follows:

$$
K_{T}^{\prime}=\left(\frac{\partial K_{T}}{\partial P}\right)_{T}=-\frac{V}{K_{T}}\left(\frac{\partial K_{T}}{\partial V}\right)_{T} .
$$

Using (22), we obtained the expression for the first pressure derivative of bulk modulus, written as

$$
\begin{aligned}
K_{T}^{\prime} & =\frac{K_{T_{0}}}{K_{T}}(1+n f)^{\frac{3}{n}+1} \\
\times & \left.\times 2+n+\left(3+4 n+\frac{4}{3} n^{2}\right) f\right] .
\end{aligned}
$$

The above expression (23) is required for the first pressure derivative of bulk modulus.

\subsection{Third-order equation of state}

To obtain the third-order equation of state (EOS), (11) has to be truncated up to the third term. Hence (11) reduces to

$$
A \cong a_{2} f^{2}+a_{3} f^{3}
$$

With the help of (14), one obtains the pressure in the form of

$$
P=\frac{1}{3 V_{0}}\left(2 a_{2} f+3 a_{3} f^{2}\right)(1+n f)^{\frac{3}{n}+1},
$$

where $a_{2}=\frac{9}{2} K_{T_{0}} V_{0}$. The undetermined third coefficient $a_{3}$ can be easily evaluated in the identical manner as for $a_{2}$.

The volume second-order derivative of pressure can be evaluated using isothermal bulk modulus and its pressure derivative. From the definition of isothermal bulk modulus

$$
\left(\frac{\partial P}{\partial V}\right)_{T}=-\frac{K_{T}}{V}
$$

we have

$$
\left(\frac{\partial^{2} P}{\partial V^{2}}\right)_{T}=\frac{K_{T}}{V^{2}}\left(K_{T}^{\prime}+1\right) .
$$

Let us apply the condition of the uncompressed state of matter, i.e., $P=0$ and $f=0$ at $V=V_{0}$. Therefore, (27) takes the following form:

$$
\left(\frac{\partial^{2} P}{\partial V^{2}}\right)_{T, P=0}=\frac{K_{T_{0}}}{V_{0}^{2}}\left(K_{T_{0}}^{\prime}+1\right)
$$

where $K^{\prime} T_{0}$ - the pressure derivative of isothermal bulk modulus at standard temperature.

Now using (25), (28) together with the expression $a_{2}=\frac{9}{2} K_{T_{0}} V_{0}$, we can easily determine the value of the third coefficient $a_{3}$. It is

$$
a_{3}=\frac{9}{2} K_{T_{0}} V_{0}\left[K^{\prime} T_{0}-(n+2)\right] .
$$

Substituting the coefficient $a_{2}$ and $a_{3}$ by (19) and (29), respectively, in pressure and volume, then (25) can be written as

$$
\begin{aligned}
P & =3 f K_{T_{0}}(1+n f)^{\frac{3}{n}+1} \\
& \times\left\{1+\frac{3 f}{2}\left[K^{\prime} T_{0}-(n+2)\right]\right\} .
\end{aligned}
$$

The second term in the curly bracket shows up due to the truncation of the Helmholtz free energy to the third-order term. The type of the curly bracket can be attributed to the considered Eulerian finite strain (8). The third-order equation of state (30) becomes indistinguishable from the secondorder equation of state $(20)$ when $K^{\prime} T_{0}=n+2$. In other words, if $K^{\prime} T_{0}=0$, the third-order equation of state (30) differs from (20).

In order to evaluate the expression for isothermal bulk modulus for third-order, the approach is identical as in (21) when the definition (17) is used. Therefore, one gets

$$
\begin{aligned}
& K_{T}=K_{T_{0}}(1+n f)^{\frac{3}{n}+1}\{1+(3+2 n) f \\
& \left.+\frac{3}{2}\left[K_{T_{0}}^{\prime}-(n+2)\right]\left[2 f+3(n+1) f^{2}\right]\right\},
\end{aligned}
$$

i.e., the expression for isothermal bulk modulus for third-order in terms of the Eulerian finite strain $f$. Note that (31) is identical to isothermal bulk modulus (21) for second-order equation of state (EOSs) if $K^{\prime} T_{0}=n+2$. In other words, if $K_{T_{0}}^{\prime}=0$, (31) should differ from (21).

Using (22), the first pressure derivative of bulk modulus with respect to the third-order equation of state can be determined. Thus,

$$
\begin{aligned}
& K_{T}^{\prime}=\frac{K_{T_{0}}}{K_{T}}(1+n f)^{\frac{3}{n}+1}\left\{K_{T_{0}}^{\prime}+\frac{(2 n+3)^{2}}{3} f\right. \\
& +(5 n+6)\left[K_{T_{0}}^{\prime}-(n+2)\right] f \\
& \left.\quad+\frac{9}{2}(n+1)^{2}\left[K_{T_{0}}^{\prime}-(n+2)\right] f^{2}\right\}
\end{aligned}
$$

represents the expression for the first pressure derivative of bulk modulus with respect to the thirdorder equation of state.

\section{Results and discussion}

The above mentioned equations, (30)-(32), are the expressions for the third-order equation of state, isothermal bulk modulus and its pressure derivative, respectively, obtained using the $n$-th power of edge length change by compression. The other equations of state can be derived by choosing the proper values of $n$ such that $n=1,1.5,2$, and 3 . Referring to these equations of state, we can also get the expression for isothermal bulk modulus and its pressure derivative. 
Case I. If $n=1$, then the $P-V$ relation (EOS), the expression for isothermal bulk modulus and its pressure derivative are

$$
\begin{aligned}
& P=3 f K_{T_{0}}(1+f)^{4}\left[1+\frac{3 f}{2}\left(K_{T_{0}}^{\prime}-3\right)\right] \\
& K_{T}=K_{T_{0}}(1+f)^{4} \\
& \quad \times\left[1+5 f+\frac{3}{2}\left(K_{T_{0}}^{\prime}-3\right)\left(2 f+6 f^{2}\right)\right] \\
& K_{T}^{\prime}=\frac{K_{T_{0}}}{K_{T}}(1+f)^{4}\left[K_{T_{0}}^{\prime}+\frac{25}{3} f\right. \\
& \left.\quad+11\left(K_{T_{0}}^{\prime}-3\right) f+18\left(K_{T_{0}}^{\prime}-3\right) f^{2}\right] .
\end{aligned}
$$

The result for $n=1$ represents the pressure-volume relation for the third-order equation of state. This is similar to the third-order first power Eulerian EOS, known as the Bardeen EOS. The results given by (34) and (35) represent their expression for isothermal bulk modulus and its pressure derivative, respectively.

Case II. If $n=1.5$, then the $P-V$ relation (EOS), the expression for isothermal bulk modulus and its pressure derivative are

$$
\begin{aligned}
& P=3 f K_{T_{0}}\left(1+\frac{3}{2} f\right)^{3}\left[1+\frac{3 f}{2}\left(K_{T_{0}}^{\prime}-\frac{7}{2}\right)\right] \\
& K_{T}=K_{T_{0}}\left(1+\frac{3}{2} f\right)^{3} \\
& \quad \times\left[1+6 f+\frac{3}{2}\left(K_{T_{0}}^{\prime}-\frac{7}{2}\right)\left(2 f+\frac{15}{2} f^{2}\right)\right], \\
& K_{T}^{\prime}=\frac{K_{T_{0}}}{K_{T}}\left(1+\frac{3}{2} f\right)^{3}\left[K_{T_{0}}^{\prime}+12 f\right. \\
& \left.\quad+\frac{27}{2}\left(K_{T_{0}}^{\prime}-\frac{7}{2}\right) f+\frac{225}{8}\left(K_{T_{0}}^{\prime}-\frac{7}{2}\right) f^{2}\right]
\end{aligned}
$$

The result given by (36) represents the pressurevolume relation for the third-order equation of state when $n=1.5$. In turn, (37) and (38) represent the expression for isothermal bulk modulus and its pressure derivative, respectively.

Case III. If $n=2$, then the $P-V$ relation (EOS), the expression for isothermal bulk modulus and its pressure derivative are

$$
\begin{aligned}
& P=3 f K_{T_{0}}(1+2 f)^{5 / 2}\left[1+\frac{3 f}{2}\left(K^{\prime} T_{0}-4\right)\right] \\
& K_{T}=K_{T_{0}}(1+2 f)^{5 / 2} \\
& \quad \times\left[1+7 f+\frac{3}{2}\left(K_{T_{0}}^{\prime}-4\right)\left(2 f+9 f^{2}\right)\right] \\
& K_{T}^{\prime}=\frac{K_{T_{0}}}{K_{T}}(1+2 f)^{5 / 2}\left[K^{\prime} T_{0}+\frac{49}{3} f\right. \\
& \left.\quad+16\left(K_{T_{0}}^{\prime}-4\right) f+\frac{81}{2}\left(K_{T_{0}}^{\prime}-4\right) f^{2}\right] .
\end{aligned}
$$

It is evident from (39) that the case for $n=2$ indicates the pressure-volume relation. This is parallel to the Birch-Murnaghan EOS of third-order. The two remaining results, (40) and (41), illustrate the expression for isothermal bulk modulus and its pressure derivative, respectively.

Case IV. If $n=3$, then the $P-V$ relation (EOS), the expression for isothermal bulk modulus and its pressure derivative are

$$
\begin{aligned}
& P=3 f K_{T_{0}}(1+3 f)^{2}\left[1+\frac{3 f}{2}\left(K_{T_{0}}^{\prime}-5\right)\right], \\
& K_{T}=K_{T_{0}}(1+3 f)^{3} \\
& \quad \times\left[1+9 f+\frac{3}{2}\left(K_{T_{0}}^{\prime}-5\right)\left(2 f+12 f^{2}\right)\right], \\
& K_{T}^{\prime}=\frac{K_{T_{0}}}{K_{T}}(1+3 f)^{2}\left[K_{T_{0}}^{\prime}+27 f\right. \\
& \left.\quad+21\left(K_{T_{0}}^{\prime}-5\right) f+72\left(K_{T_{0}}^{\prime}-5\right) f^{2}\right] .
\end{aligned}
$$

The result given by (42) provides the pressurevolume relation identical to the third-power Eulerian EOS of third-order. In turn, (43) and (44) define the expression for isothermal bulk modulus and its pressure derivative, respectively. It is evident from the above derivations that the proposed formulation is capable of producing the most prominent equation of state for the various values of $n$. For example, (i) for $n=1$, the first-power Eulerian EOS (the Bardeen EOS), (ii) for $n=2$, the BirchMurnaghan EOS, and (iii) for $n=3$, the thirdpower Eulerian EOS.

The method of analysis of the new equation of state is based on the Eulerian finite strain formula applying the $n$-th power of edge length change by compression which is described under theoretical analysis (Sect. 2). The second-order equation of state (Sect. 2.1) and the third-order equation of state (Sect. 2.2) illustrate the evaluation of isothermal bulk modulus and its pressure derivative. To test the validity of the proposed work, we use (36)-(44) to compute the $P-V$ relation (EOS), isothermal bulk modulus and its pressure derivative. The results are obtained for alkaline earth oxides viz. $\mathrm{MgO}, \mathrm{CaO}$, and plotted. The present formulation shows the comparison for the values of $n=1.5$ with BM EOS and TP Eulerian EOS. These values obtained are also compared with other theoretical models as well as the available experimental results. The input parameters for formulation for alkaline earth oxides have been shown in Table I $[2,15,16]$.

Figure 1 shows the dependence of the finite strain vs compression for different values of $n$. The finite strain increases almost similarly up of the compression value of $V / V_{0}=0.8$ for all values of $n$. However, as $V / V_{0} \rightarrow$, the finite strains sharply increases and then diverges to infinity. As a result, the Helmholtz free energy and its volume derivative, i.e., pressure, also increase to infinity 


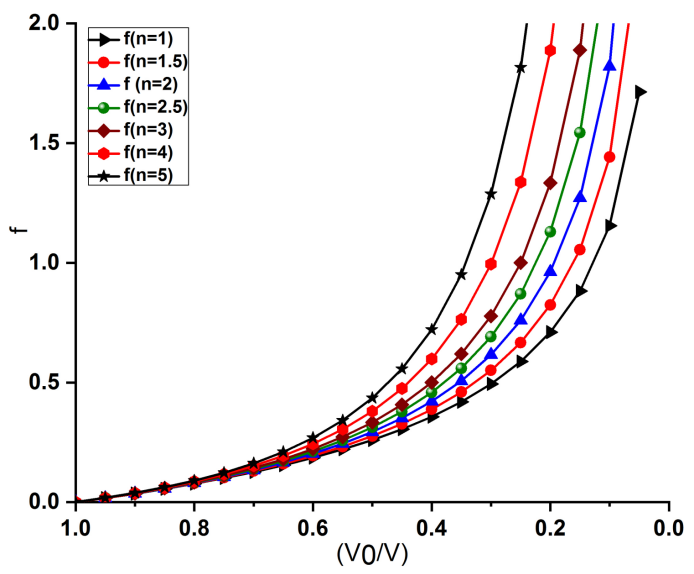

Fig. 1. Comparison of the Eulerian finite strain $(f)$ at different values of $n$ derived by the expansion of the $n$-th powered length as a function of compression $\left(V / V_{0}\right)$.

TABLE I

The input parameters bulk moduli $\left(K_{T}\right)$ (in GPa) and pressure derivative of bulk moduli $\left(K_{T}^{\prime}\right)$ (dimensionless) at ambient pressure $(P=0)$ and room temperature $(300 \mathrm{~K})$ recorded in respective reference papers.

\begin{tabular}{c|c|c}
\hline \hline Parameters & $\mathrm{MgO}$ & $\mathrm{CaO}$ \\
\hline$K_{T}$ & $161.6[2]$ & $110.6[2]$ \\
$K_{T}^{\prime}$ & $4.13[15]$ & $4.05[16]$
\end{tabular}

when $V / V_{0} \rightarrow 0$. It is also evident that different values of $n$ from 1 to 5 attained the finite value of finite stain at $V / V_{0} \rightarrow 0.05$ using the Eulerian scheme. Figure 1 indicates that the function of compression $\left(\frac{V}{V_{0}}\right)$ decreases as the value of $n$ increases.

The pressure has been computed at different isothermal compression ranging from 1 to 0.6 at $300 \mathrm{~K}$ for $\mathrm{MgO}$ and $\mathrm{CaO}$, with the use of (36), (39), and (42). The results of computations are shown in Fig. 2. We have obtained a similar trend as compression $\left(\frac{V}{V_{0}}\right) \rightarrow 0$ and pressure $P \rightarrow \infty$ with respect to the present EOS for the values 1.5, the Birch-Murnaghan EOS and third-power Eulerian EOS. These equations are equivalent to the basic thermodynamic conditions as well as the Stacey criteria. The results concerning the case of $\mathrm{MgO}$ are shown in Fig. 2a. There, the percentage deviation at compression value $\left(V / V_{0}\right)=0.7197$ is $4.376,4.374$, and 8.334, respectively, for the present model (36), the Birch-Murnaghan EOS (39), and third-power Eulerian EOS (42). Karki et al. [6] reported 9.173, while Oganov and Dorogokupets [8] obtained the percentage deviation value of 7.35. Thus, one can state that the present equation of state used for the value $n=1.5$ shows a good agreement with the experimental results [17] when compared to the third-power Eulerian, Karki and Oganov models $[6,8]$. It is also the evidence of the good consistency of the present model.
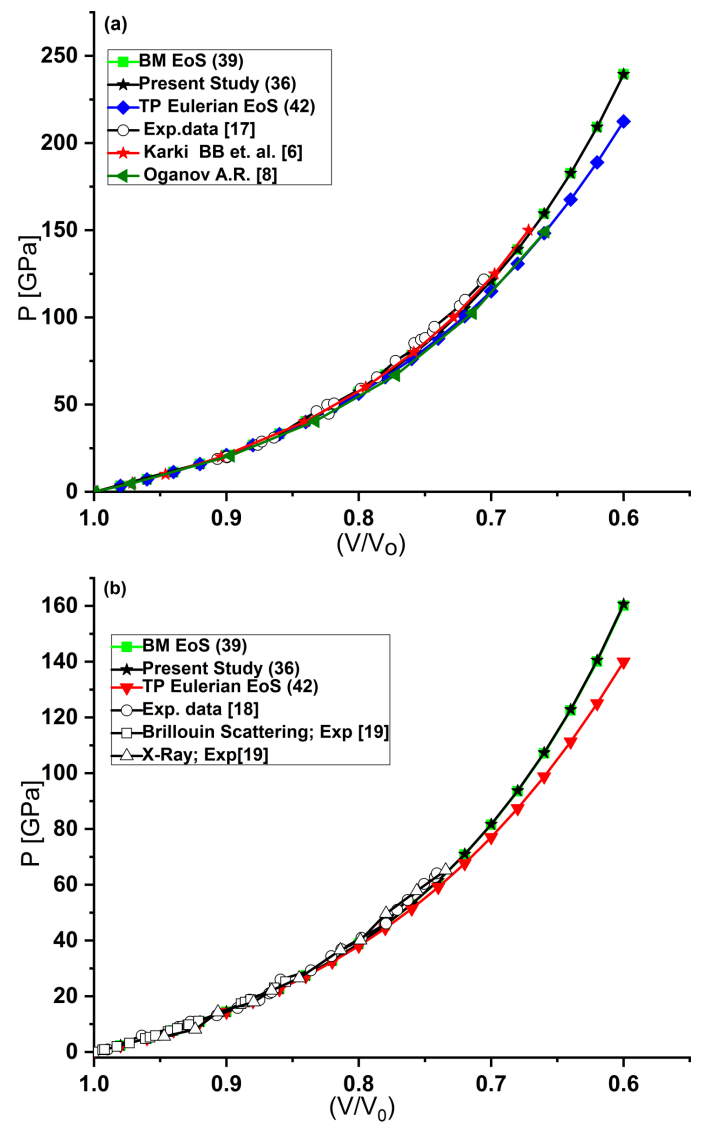

Fig. 2. Compression curves for different values of $n$ of the equation of state for the $n$-th powered length. The figures compare the calculated results and other models (filled symbols) with experimental data (open symbols) (a) $\mathrm{MgO}$, (b) $\mathrm{CaO}$.

The results concerning the case of $\mathrm{CaO}$ are shown in Fig. 2b. There, the percentage deviations at compression value $\left(V / V_{0}\right)=0.741$ is 4.745 for the present model (36), 4.865 for the BirchMurnaghan EOS (39) and 8.4 for third-power Eulerian EOS (42). The $P-V$ dependence for $\mathrm{CaO}$ and the results obtained by the present model (36) match well with experimental results [18, 19], when compared to the Birch-Murnaghan EOS and third-power Eulerian EOS. In fact, the third-power Eulerian EOS diverged more from experimental results $[18,19]$.

The expression for pressure dependence of isothermal bulk modulus has been obtained by using (31). The present work estimates pressure ranges of these two oxides which are wider than the pressure ranges related to the B1-type phases and stable enough to investigate the systematic of elasticity of alkaline earth oxides.

The bulk moduli $K_{T}$ for the present model, the Birch-Murnaghan EOS and the third-power Eulerian EOS have been obtained for the values of $n=1.5,2$, and 3 . The result obtained for pressure dependence of isothermal bulk modulus has been represented by (37), (40), and (43). The derived 

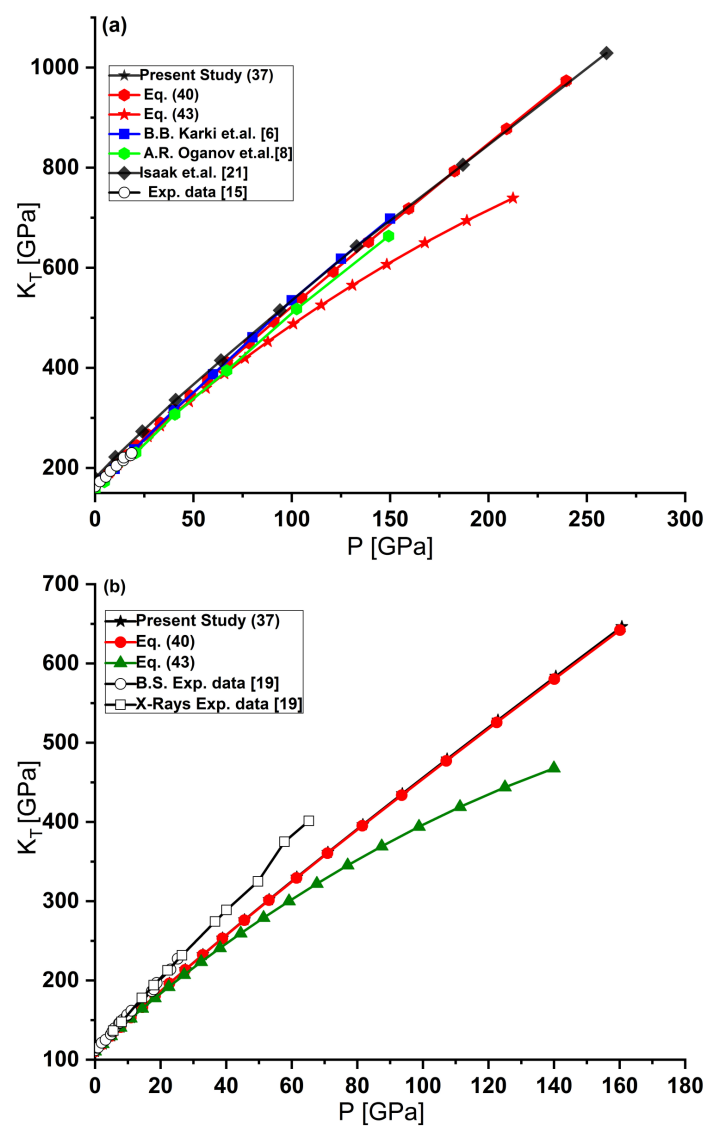

Fig. 3. Pressure dependence of bulk modulus: (a) $\mathrm{MgO}$, (b) $\mathrm{CaO}$. The filled and color symbols are calculated results and other models for $K_{T}$. Open symbols show experimental data.

expression of $K_{T}$ corresponding to the above equation of states has been applied to $\mathrm{MgO}$ and $\mathrm{CaO}$. The calculated bulk moduli as functions of pressure are shown in Fig. 3. It is interesting to note that the value of $K_{T}$ increases with the pressure growth. The present results (37) agree well with PWPP LDA results [6], ab initio [20] and quite well with DFT GGA results [8] in the case of $\mathrm{MgO}$. All theoretical calculations, i.e., (37), (40), PWPP LDA [6], DFT GGA [8], ab initio [20], match with each other and experimental results for the pressure value of $20 \mathrm{GPa}$. However, (43) (TP Eulerian EOS) does not correspond to the theoretical calculations [6, 8, 20] as well as experimental results [15].

In the case of $\mathrm{CaO}$, the present results (37) correspond well with (40) and reasonably well with experimental results [19]. However, (43) shows more deviation from experimental results measured by Speziale et al. [19], where the method of the singlecrystal Brillouin scattering to 25.2 GPa was used, together with powder X-ray diffraction to $65.2 \mathrm{GPa}$ in a radial geometry at ambient temperature in a diamond cell.

From the geophysical point of view, the first pressure derivative of the isothermal bulk modulus is a parameter that is necessary for the accurate
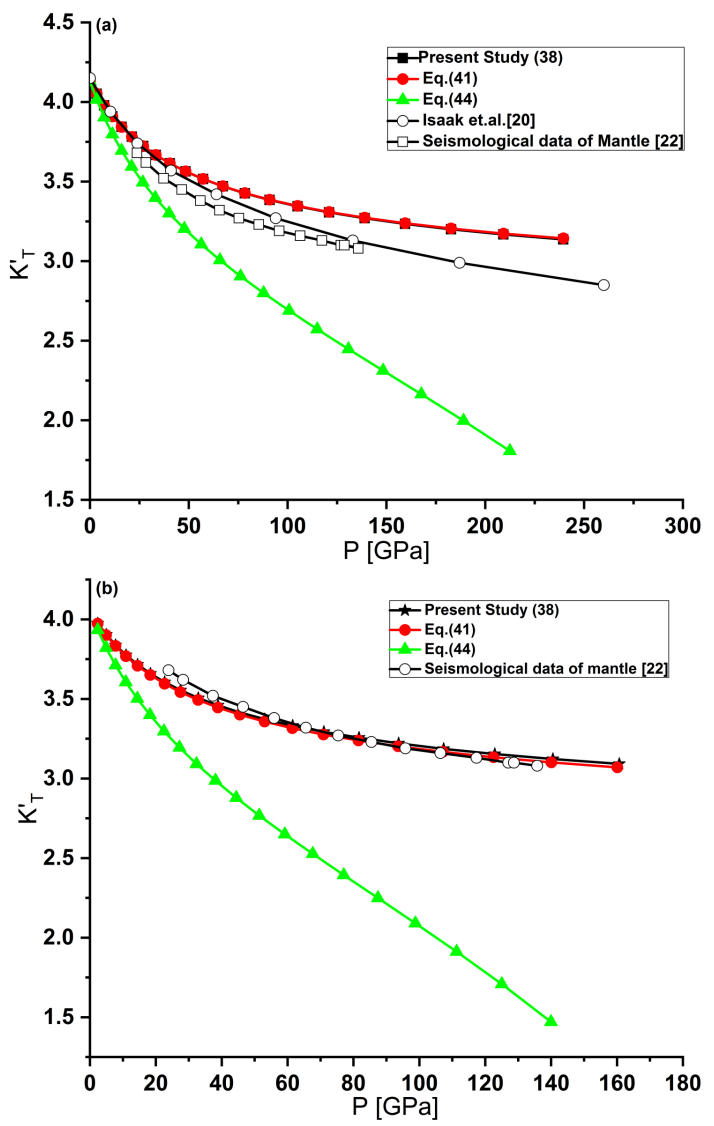

Fig. 4. Pressure derivative $K_{T}^{\prime}$ vs pressure at $300 \mathrm{~K}$ for (a) $\mathrm{MgO}$, (b) $\mathrm{CaO}$.

inversion of the seismic data into composition, structure, and texture of earth, as well as for determining the thermal properties of the deep earth [21] and the isothermal empirical equation of state of the materials in the deep earth [1].

The results for pressure derivative $K_{T}^{\prime}$ obtained from different equations, i.e., (38), (41) and (44), have been compared with other theoretical results and experiments. The obtained results are shown in Fig. 4a and $4 \mathrm{~b}$ for $\mathrm{MgO}$ and $\mathrm{CaO}$, respectively. Figure 4 shows the decrease of $K_{T}^{\prime}$ values as the pressure increases to $100 \mathrm{GPa}$. After that, the $K_{T}^{\prime}$ values start to decrease very slowly with increasing pressure. In turn, (44), based on TP Eulerian EOS, showed a strong decrease with increasing pressure as compared to all theoretical models and experimental results.

The results for alkaline earth oxides in Fig. 4 exhibit similar trends and in fact correspond better with the seismological data [22] reported by Stacey and Davis. Our results confirm the major role of alkaline earth oxides and magnesiowustite in the composition of earth's mantle and the seismic heterogeneity that exists throughout the lower mantle of earth. It is more likely that this heterogeneity is due to a combination of chemical, thermal and phase change effects [23]. Comparisons between seismological data and the elastic properties 
of candidate mantle minerals and assemblages are the only way to extract information regarding the composition and mineralogy of the mantle from the rich seismological database [6]. The elasticity of minerals also yields substantial insight into the nature of bonding.

Thus, the derived formulation is efficient to predict the elastic properties under the varying conditions of pressure. Due to the accessibility of the method, it can be implemented to other complicated minerals having geophysical importance and applications.

\section{Conclusions}

The results from the proposed EOSs are very satisfactory in comparison to other theoretical models $[5,8,9]$ and match well with experimental results $[15-20]$. The main feature of this model is that less input parameters are required which are easily available in experimental studies. The present study is capable of producing various equations of state. These models may be frequently employed as the thermal equation of states of earth's minerals. It can be exploited for various advanced quantum methods and simulations at the pressure and temperature of earth's interior. The present study follows the basic laws of thermodynamics with regard to expression at high-pressure. It allows extrapolation to regions for which experimental data are not available. These models may, therefore, be useful for future planning of high-pressure experiments on the compression behavior of earth forming minerals, solids, nanomaterials, etc.

\section{Acknowledgments}

This research program was supported by Uttar Pradesh Government grant under the scheme "Research and Development". Authors are grateful to Dr. Pushpa Kashyap, Principal, Dr. B.R. Ambedkar Government Degree College, Mainpuri (UP), India, for facilitating the necessary amenities. We are also grateful to the reviewer for his valuable and constructive suggestions which have been very useful in revising this manuscript.

\section{References}

[1] O.L. Anderson, Equations of State of Solids for Geophysics and Ceramic Science, Oxford University Press, New York 1995.

[2] O.L. Anderson, D.G. Isaak, Mineral Physics and Crystallography: A Handbook of Physical Constants, Vol. 2, UCLA/American Geophysics Union, Los Angeles (CA) 1995, p. 64.

[3] D.G. Isaak, I. Ohno, Phys. Chem. Mineral. 30, 430 (2003).
[4] S.V. Sinogeikin, J.D. Bass, T. Katsura, Phys. Earth Planet. Inter. 136, 41 (2003).

[5] S.P. Singh, S. Gupta, S.C. Goyal, Physica B 391, 307 (2007).

[6] B.B. Karki, G.J. Ackland, J. Crain, J. Phys. Condens. Matter 9, 8579 (1997).

[7] B.B. Karki, R.M. Wentzcovitch, S. De Gironcoli, S. Baroni, Phys. Rev. B 61, 8793 (2000).

[8] A.R. Oganov, P.I. Dorogokupets, Phys. Rev. B 67, 224110 (2003).

[9] T. Tsuchiya, K. Kawamura, J. Chem. Phys. 114, 10086 (2001).

[10] S.P. Singh, Int. J. Mod. Phys. Conf. Series 22, 397 (2013).

[11] C.R. Bina, G.R. Helffrich, Annu. Rev. Earth Planet. Sci. 20, 527 (1992).

[12] O.L. Anderson, H. Oda, D. Isaak, Geophys. Res. Lett. 19, 1987 (1992).

[13] T.S. Duffy, R.J. Hemley, H.K. Mao, Phys. Rev. Lett. 74, 1371 (1995).

[14] H. Baltache, R. Khenata, M. Sahnoun, M. Driz, B. Abbar, B. Bouhafs, Physica B 344, 334 (2004).

[15] S.V. Sinogeikin, J.D. Bass, Phys. Rev. B 59, R14141 (1999).

[16] Z.P. Chang, E.K. Graham, J. Phys. Chem. Solids 38, 1355 (1977).

[17] S.P. Marsh, LASL Shock Hugoniot Data, Vol. 5, University of California Press, 1980.

[18] J.F. Mammone, H.K. Mao, P.M. Bell, Geophys. Res. Lett. 8, 140 (1981).

[19] S. Speziale, S.R. Shieh, T.S. Duffy, J. Geophys. Res. 111, B02203 (2006).

[20] D.G. Isaak, R.E. Cohen, M.J. Mehl, J. Geophys. Res. 95, 7055 (1990).

[21] T.S. Duffy, D.L. Anderson, J. Geophys. Res. 94, 1895 (1989).

[22] F.D. Stacey, P.M. Davis, Phys. Earth Planet Inter. 142, 137 (2004).

[23] J.H. Woodhouse, A.M. Dziewonski, Philos. Trans. R. Soc. Lond. A 328, 291 (1989). 